\title{
Brain Stroke as a Post-0perative Lethal Complication of a Bilateral Chronic Subdural Hematoma: A Case Report
}

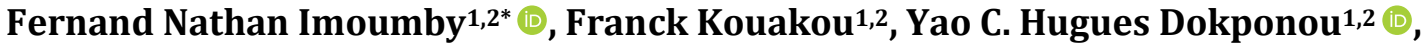 \\ Abad Cherif El Asri' ${ }^{1,2}$, Miloud Gazzaz ${ }^{1,2}$ \\ ${ }^{1}$ Mohammed V Military Teaching Hospital, Rabat, Morocco \\ ${ }^{2}$ Faculty of Medicine and Pharmacy, Mohammed V University, Rabat, Morocco \\ Email: `ifernandnathan@gmail.com, kouakouloukoufranck777@gmail.com, dokponou2407@gmail.com, \\ abad20031@gmail.com,gmiloneuro@yahoo.fr
}

How to cite this paper: Imoumby, F.N., Kouakou, F., Dokponou, Y.C.H., El Asri, A.C. and Gazzaz, M. (2021) Brain Stroke as a Post-Operative Lethal Complication of a Bilateral Chronic Subdural Hematoma: A Case Report. Open Journal of Modern Neurosurgery, 11, 122-127.

https://doi.org/10.4236/ojmn.2021.112015

Received: March 18, 2021

Accepted: April 26, 2021

Published: April 29, 2021

Copyright $\odot 2021$ by author(s) and Scientific Research Publishing Inc. This work is licensed under the Creative Commons Attribution International License (CC BY 4.0).

http://creativecommons.org/licenses/by/4.0/

\begin{abstract}
Chronic subdural hematoma (CSDH) is a common neurosurgical condition that is frequently encountered in neurosurgery practice. Its etiologies are dominated by traumatic head injury, and clinical presentations are various. The prognosis is generally good, but complications can be seen. Some of these complications can lead to the death of the patient. We report the rare case of a 70-year-old man admitted to the emergency room for the management of bilateral CSDH revealed by severe headache, vomiting, and loss of consciousness. He underwent a successful evacuation of both hematomas but had a stroke in both posterior cerebral artery territories, which was lethal 72 hours after surgery. We present and try to find an explanation for this exceptional post-operative complication of $\mathrm{CSDH}$.
\end{abstract}

\section{Keywords}

Brain Stroke, Complication, Subdural Hematoma, Case Report

\section{Introduction}

Chronic subdural hematomas (CSDH) are a common neurological surgery condition. Primary management for the symptomatic lesion usually entails surgical intervention. There is controversy regarding ideal modality selection among twist drill craniostomy, burr hole craniostomy, and craniotomy [1]. Known to be often the result of a traumatic subdural effusion as authors described it in the literature [2], other causes like neurosurgical operations, alcoholism, anticoagu- 
lant drugs, or coagulopathy can be encountered. In general, the outcome is good but complications such as acute subdural hematoma, tension pneumocephalus, and more rarely subdural empyema, and brain infarction as found by Mori et al. [3] can occur. Brain stroke in that condition is potentially lethal. We report and discuss the onset hypothesis of a rare case of a 70-year-old man with no past medical history, who underwent an evacuation of a bilateral CSDH and presented in the post-operative period a brain infarction in right and left posterior cerebral artery territories.

\section{Case Presentation}

A 70-year-old man with a history of intermittent and frequent headaches for the past two (2) months, was admitted to the emergency room for worsening of the headache associated with vomiting. There were no complaints about blurry vision. The patient denies any recent history of head trauma, hypertension, and diabetes. He didn't smoke or consume alcohol. At the admission, the patient suddenly lost consciousness with a Glasgow Coma Scale (GCS) of 6/15 without sensory-motor palsy or fever. He was transferred to the intensive care unit for initial conditioning with monitoring of vital parameters after sedation and intubation. The pupils were poorly responsive but brainstem reflexes were present.

\subsection{Investigation}

A brain CT-scan was performed and found a bulky bilateral and hemispheric chronic subdural hematoma with an acute bleeding area on the left side (Figure 1). Laboratory findings were normal as well as the electrocardiogram and the chest X-ray.

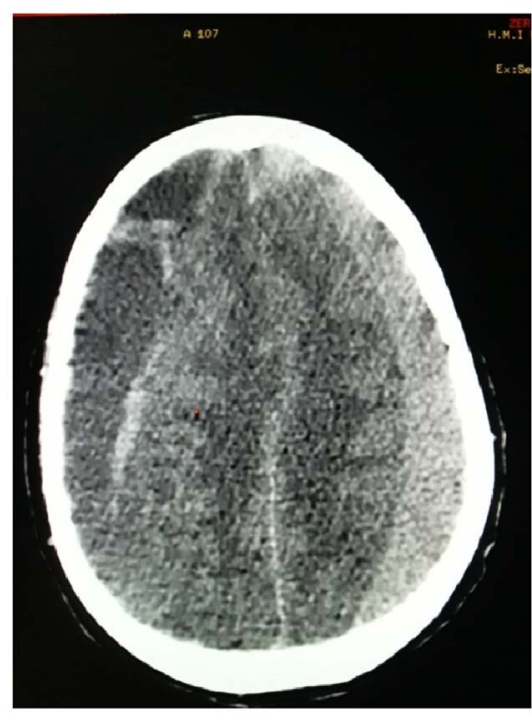

(a)

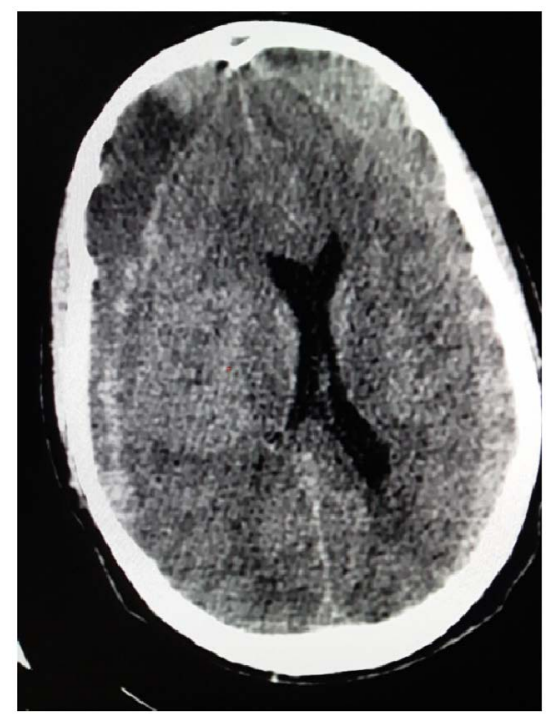

(b)

Figure 1. Pre-operative CT-scan showing Bilateral and hemispheric CSDH with subacute re-bleeding on the left side. We can note the left shift of midline (a) and the compression on the right ventricle (b) due to right hematoma mass effect. 


\subsection{Management and Outcome}

Both hematomas were evacuated emergently through two burr holes on both sides under general anesthesia. Dark old blood was removed and the subdural space was washed with warm $0.9 \%$ saline solution until all the hematoma is out. No evidence of active bleeding was found. The operative incision was then closed on top of Kehr drainage. There was no incident or difficulty during surgery. Vital signs did not change and arterial blood pressure did not rise during surgery. The patient was then brought back to the intensive care unit. Note that despite his advanced age, postoperatively the patient did not receive a prophylactic anticoagulation for antithrombotic purposes, to avoid re-bleeding through the drains that were in place. Twenty-four (24) hours later a brain CT-scan was performed because of a delayed awakening of the patient, which showed extensive ischemia in both posterior cerebral artery territories (Figure 2). The etiological assessment, cardiac, endovascular and metabolic was normal, and the only favoring factor identified was old age. The patient didn't receive any management related to this stroke because of his poor prognosis. The patient died in the intensive care unit 72 hours after surgery.

\section{Discussion}

Chronic subdural hematoma (CSDH) is known to have a good prognosis with a relatively simple surgical intervention. However, some complications can occur. Brain infarction after subdural chronic hematoma removal is very rare. There are several possible explanations for postoperative stroke in both vascular territories of the posterior cerebral artery. Firstly, the patient presented per operatively a termino-basilar thrombosis realizing one form of top of the basilar artery syndrome described by Requena et al. [4]. That hypothesis may be sustained by the age of the patient, even if he had no clinical manifestation until the day surgery was performed. Secondly, according to the acute deterioration of the consciousness, and the context of intensive headaches, it may be acute intracranial hypertension which resulted in a bilateral temporal lobe herniation causing

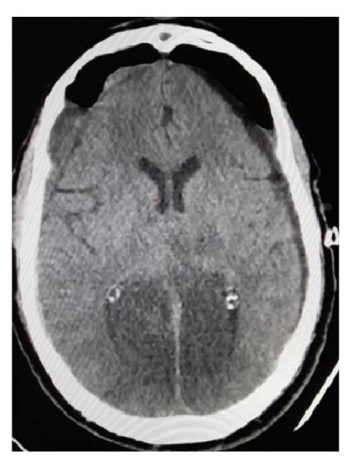

(a)

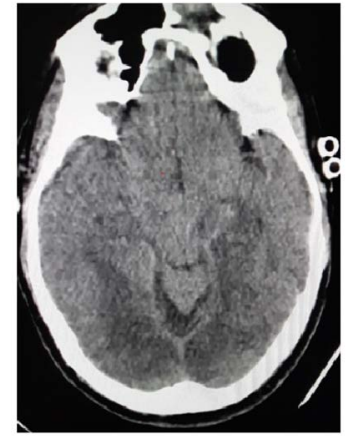

(b)

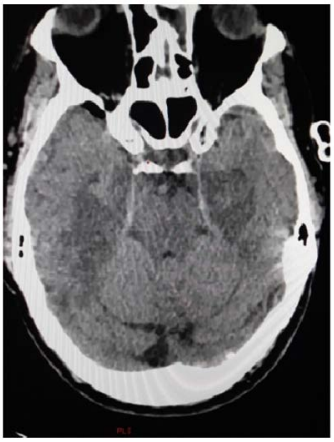

(c)

Figure 2. Post-operative CT-scan showing extensive infarction in the left and right territories of posterior cerebral artery ((a), (b) and (c)), with bilateral frontal pneumocephalus and incomplete evacuation of the hematoma predominant in the right side (a). 
crushing of the two posterior cerebral arteries and then the stroke in their both cerebral areas. In this case, ischemia was not seen in the preoperative CT-scan because it was done most probably during the early stage of the brain infarction. A Diffusion MRI sequence would be appropriate to see the stroke at that moment.

However, the most important factor, as discussed by several authors [5] [6] is the spontaneous intracranial hypotension (SIH) which would result in extreme brain sagging and could lead to anatomical distortion of the diencephalon and brainstem resulting in coma and secondarily in brain stroke. The SIH occurs most often after a lumbar puncture, craniotomy, or spinal surgery and is due to CSF leakage from a dural tear. Rarely, it may occur as a consequence of severe dehydration, diabetic ketoacidosis, or uremia [7]. The diagnosis is suspected in front of orthostatic headaches, however, cases of non-positional headache had been reported by authors as Schievenk et al. [8], our patient would be in the non-positional headache group. The diagnosis is confirmed by the evident findings of CSF leaking by CT-myelogram or spinal MRI. Its treatment is based on using the blood patch, and when it is associated with chronic subdural hematoma, there is no codified management. Nevertheless, several authors wrote on the subject [5] [6] [9] [10] [11]. According to us, we propose, as Akhaddar et al. in the general management of $\mathrm{CSDH}$ and to prevent intracranial rebleeding outcomes [12], to perform a very safe evacuation of subdural hematoma making it slowly and gradually through burr hole craniostomy or using twist drill craniostomy.

\section{Conclusion}

Brain stroke as a postoperative complication of chronic subdural hematoma is a very rare entity. So its occurrence may give us to recall spontaneous intracranial hypotension. It is necessary to think about this, in front of every patient presenting with an orthostatic or non-positional CSDH headache. We need to offer the best management to the patients with these conditions because of the gravity attached to the outcome of the cerebral ischemia.

\section{Ethics and Reporting Guidelines}

Informed consent and verbal permission were obtained from the family of the patient prior to the submission of this article. Also, this article respects both the Consensus-based Clinical Case Reporting Guideline and the Recommendations for the Conducting, Reporting, Editing, and Publication of Scholarly Work in Medical Journals [13] [14].

\section{Author Contributions}

Fernand Nathan Imoumby: Conceptualization, Writing an original draft, \& editing. Loukou Franck Kouakou: Writing, review \& editing. Yao Christian Hugues Dokponou: Writing, review \& editing. Abad Cherif El Asri: review \& 
editing. Miloud Gazzaz: Supervision, review, \& Validation.

\section{Conflicts of Interest}

The authors declare not having any conflict of interest in this case report.

\section{References}

[1] Abecassis, I.J. and Kim, L.J. (2017) Craniotomy for Treatment of Chronic Subdural Hematoma. Neurosurgery Clinics of North America, 28, 229-237. https://doi.org/10.1016/j.nec.2016.11.005

[2] Murata, K. (1993) Chronic Subdural Hematoma May Be Preceded by Persistent Traumatic Subdural Effusion. Neurologia Medico-Chirurgica (Tokyo), 33, 691-696. https://doi.org/10.2176/nmc.33.691

[3] Mori, K. and Maeda, M. (2001) Surgical Treatment of Chronic Subdural Hematoma in 500 Consecutive Cases: Clinical Characteristics, Surgical Outcome, Complications, and Recurrence Rate. Neurologia Medico-Chirurgica (Tokyo), 41, 371-381. https://doi.org/10.2176/nmc.41.371

[4] Esquinas Requena, J.L., Gonzalez Ruiz, L., Fernandez Martinez, N., Parras Garcia de Leon, N. and Gil Moreno, J. (2017) Descripcion de un sindrome del top de la basilar. Una entidad neurologica pocofrecuente. Apunt Cienc, 7, 21-24.

[5] Schievink, W.I. (2013) Stroke and Death Due to Spontaneous Intracranial Hypotension. Neurocritical Care, 18, 248-251. https://doi.org/10.1007/s12028-012-9800-3

[6] Dhillon, A.K., Rabinstein, A.A. and Wijdicks, E.F.M. (2010) Coma from Worsening Spontaneous Intracranial Hypotension after Subdural Hematoma Evacuation. Neurocritical Care, 12, 390-394. https://doi.org/10.1007/s12028-009-9323-8

[7] Renowden, S.A., Gregory, R., Hyman, N. and Hilton-Jones, D. (1995) Spontaneous Intracranial Hypotension. Journal of Neurology, Neurosurgery \& Psychiatry, 59, 511-515. https://doi.org/10.1136/jnnp.59.5.511

[8] Schievink, W.I. and Smith, K.A. (1998) Nonpositional Headache Caused by Spontaneous Intracranial Hypotension. Neurology, 51, 1768-1769.

https://doi.org/10.1212/WNL.51.6.1768

[9] Matsuoka, K., Nakai, E., Kawanishi, Y., Kadota, T., Fukuda, H. and Ueba, T. (2020) Acute Deterioration in a Patient with Bilateral Chronic Subdural Hematomas Associated with Intracranial Hypotension Treated with an Epidural Blood Patch. World Neurosurgery, 141, 331-334. https://doi.org/10.1016/j.wneu.2020.05.254

[10] Zou, L.H., Li, G.C., Zhao, J.C., Zhang, Y. and Hou, K. (2020) Management of Spontaneous Cerebrospinal Fluid Hypovolemia-Associated Massive Chronic Subdural Hematoma with Reinforced Restriction of Physical Activity: Report of Three Cases. The Journal of International Medical Research, 48, 1-8. https://pubmed.ncbi.nlm.nih.gov/33213246/ https://doi.org/10.1177/0300060520969321

[11] Wan, Y., Xie, J., Xie, D., Xue, Z., Wang, Y. and Yang, S. (2016) Clinical Characteristics of 15 Cases of Chronic Subdural Hematomas Due to Spontaneous Intracranial Hypotension with Spinal Cerebrospinal Fluid Leak. Acta Neurologica Belgica, 116, 509-512. https://doi.org/10.1007/s13760-016-0597-2

[12] Akhaddar, A., Ajja, A., Elmostarchid, B. and Boucetta, M. (2008) Combined Epidural and Intracerebral Hematomas after Evacuation of Bilateral Chronic Subdural Hematoma. Neurochirurgie, 54, 728-730. https://doi.org/10.1016/j.neuchi.2008.09.001

[13] Gagnier, J.J., Kienle, G., Altman, D.G., Moher, D., Sox, H., Riley, D., et al. (2013) 
The CARE Guidelines: Consensus-Based Clinical Case Reporting Guideline Development. Case Reports, 2013, Article ID: bcr2013201554.

https://doi.org/10.1136/bcr-2013-201554

[14] International Committee of Medical Journal Editors (2020) Recommendations for the Conducting, Reporting, Editing, and Publication of Scholarly Work in Medical Journals. ICMJE 2019. http://www.icmje.org/recommendations/ 